\title{
Motion Planning of Two Stacker Cranes in a Large-Scale Automated Storage/Retrieval System*
}

\author{
Yiheng KUNG**, Yoshimasa KOBAYASHI***, Toshimitsu HIGASHI*** \\ and Jun OTA** \\ **Research into Artifacts, Center for Engineering (RACE), The University of Tokyo, \\ 5-1-5 Kashiwanoha, Kashiwa, Chiba 277-8568, Japan \\ E-mail: ota@race.u-tokyo.ac.jp \\ ***Murata Machinery, Ltd. \\ 2, Nakajima, Hashizume, Inuyama, Aichi 484-8502, Japan
}

\begin{abstract}
We propose a method for reducing the computational time of motion planning for stacker cranes. Most automated storage/retrieval systems (AS/RSs) are only equipped with one stacker crane. However, this is logistically challenging, and greater work efficiency in warehouses, such as those using two stacker cranes, is required. In this paper, a warehouse with two stacker cranes working simultaneously is proposed. Unlike warehouses with only one crane, trajectory planning in those with two cranes is very difficult. Since there are two cranes working together, a proper trajectory must be considered to avoid collision. However, verifying collisions is complicated and requires a considerable amount of computational time. As transport work in AS/RSs occurs randomly, motion planning cannot be conducted in advance. Planning an appropriate trajectory within a restricted duration would be a difficult task. We thereby address the current problem of motion planning requiring extensive calculation time. As a solution, we propose a "free-step" to simplify the procedure of collision verification and reduce the computational time. On the other hand, we proposed a method to reschedule the order of collision verification in order to find an appropriate trajectory in less time. By the proposed method, we reduce the calculation time to less than $1 / 300$ of that achieved in former research.
\end{abstract}

Key words: Motion Planning, Automated Storage/Retrieval System (AS/RS), Stacker Cranes

\section{Introduction}

An automated storage/retrieval system (AS/RS) is the backbone of an inventory management process. It is one that uses an automatic stacker crane to carry out tasks in a warehouse, such as loading, unloading, inventory, and warehouse arrangement (Fig. 1). As customer requirements have become diversified and frequently change, the role of an $\mathrm{AS} / \mathrm{RS}$ has gradually changed from a place where goods are kept for long-term storage to a temporary buffer that adjusts the balance between loading and unloading in a warehouse. Since AS/RSs are being used more frequently and the loading efficiency of warehouses has become important, we are committed to improving the efficiency of AS/RSs.

Kees conducted a survey of AS/RSs and concluded that five primary elements are required to improve the working efficiency using one crane [1]. These elements are: 
II. Storage assignment

III. Batching

IV. Sequencing of storage and retrieval requests

V. Motion planning of a stacker crane

Tompkins et al. studied the optimal layout of warehouses according to the horizontal and vertical speeds of a stacker crane [2]. In addition, Malmborg et al. studied the optimal storage assignment of warehouses [3-5], and Barrett et al. studied motion planning of a human-controlled crane [6-7]. Human-controlled cranes are capable of carrying more than one item, and the way in which the sequencing of transport is determined has much to do with the efficiency of warehouse work. Other studies on AS/RSs include the following topics: a travel time model [8], task sequencing [9-11], batching [12], interleaving of tasks [13], zone construction [14], design support system [15], throughput analysis [16], cube-per-order index [17-18], an integrative model [19], and operation management [20].

Even with all the elements optimized to design a suitable system, the efficiency is still below customer needs. Therefore, AS/RSs employing multiple stacker cranes have been considered a potential solution to this problem. The number of stacker cranes used in $\mathrm{AS} / \mathrm{RSs}$ is restricted by the mechanical conditions.

Since a stacker crane must stretch out its fork to remove a load, the fork lacks the capacity to sustain torque if the stacker crane is too far from the rack. In view of this mechanical restriction, an AS/RS using two stacker cranes is said to be appropriate in practice. Hino et al. studied the motion planning algorithm of a warehouse with two stacker cranes moving freely on their rails [21], as shown in Fig. 1. The motion planning method Hino proposed is able to derive an efficient trajectory that can avoid collision and finish transportation tasks in a short time as well. However, this method takes too much calculation time to do motion planning; as a result, it is not practical.

In this paper, we focus on reducing the calculation time for motion planning so that the method can be applied in the industrial field; in addition, we attempt to maintain efficiency while reducing the computational time.

There are two challenges for an AS/RS using two stacker cranes. The first is appropriate motion planning. Since two cranes are working simultaneously in a large-scale warehouse, overlapping paths could lead to crane collisions. Furthermore, unlike a multi-robot motion planning problem, the trajectory of a crane cannot be modified once the crane starts to move because of the mechanical restrictions. Collisions will occur if the trajectory is not properly scheduled. In addition, the quality of motion planning plays a large role in the resulting efficiency of warehouse work. The second challenge is the limited computational time for motion planning. Since inventory orders from customers always occur randomly, AS/RS must conduct motion planning to respond to the order immediately (proper response time is said to be 0.1 seconds by a $2 \mathrm{GHz}$ computer in the industrial field).

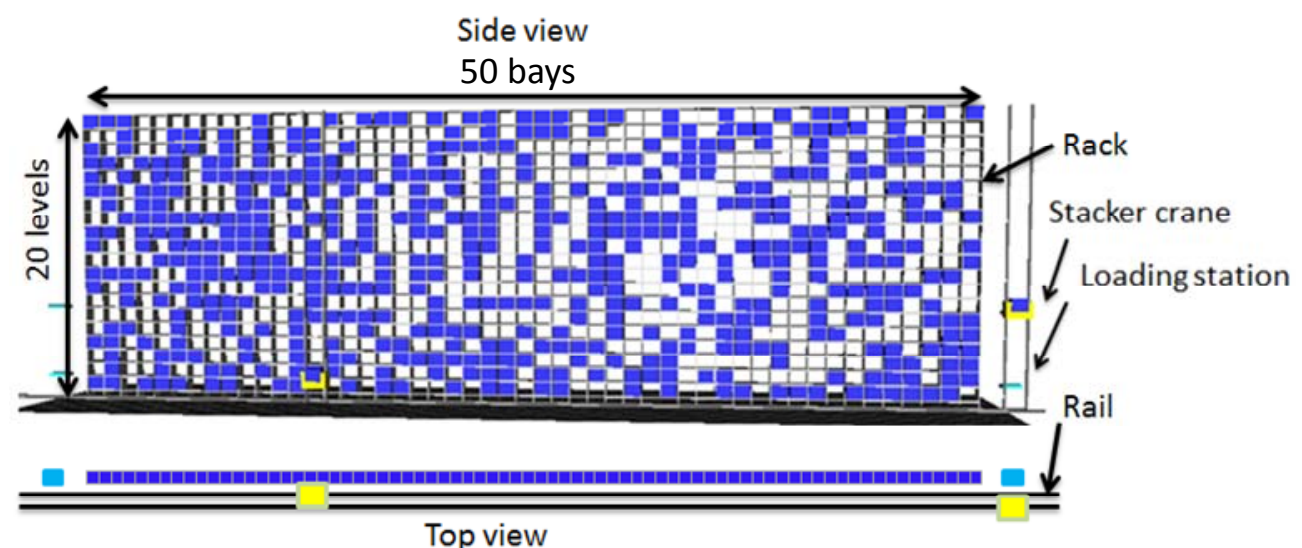

Fig. 1 an automated storage/retrieval system 


\section{Problem description and introduction to previous research}

\subsection{Problem formulation}

Although there are many kinds of warehouses, we focus exclusively on the type described below.

\subsubsection{AS/RS environment and task definition}

While there are many kinds of AS/RSs, we focus on the type shown in Fig. 1. The warehouse environment assumed in this paper is as follows:

a. The capacity of the storage rack is 256 bays on 128 levels, which is the largest practical scale ( 50 bays on 20 levels is the most popular scale in practice).

b. The size of a load is $500(\mathrm{~mm})$ by $500(\mathrm{~mm})$ by $400(\mathrm{~mm})$.

c. Two loading stations are set up at both ends of the rack.

d. Tasks are conducted simultaneously by two stacker cranes.

In this warehouse, tasks (requirements of transport) will be assigned by the controller, and each of the stacker cranes, moving freely on their rails, is used to transport the load. The stacker crane first moves to the designated location and then uses its fork to load the item and transport it to the designated destination. Once the task is finished, a new task is designated, and the stacker crane carries out the new task.

\subsubsection{Conditions for collision that should be avoided}

There are two collision patterns in warehouse work that should be avoided.

One is between stacker cranes. Due to mechanical constraints, we cannot allow for adequate distance between the rails. A crane will collide with another if its location overlaps with that of the other crane (Fig. 2(a)).

The other pattern involves the potential collision between the mast and the fork. When the outer crane outstretches its fork in order to receive a load, it will collide with the mast of the inner crane if the inner crane passes the outer crane in a horizontal direction (Fig. 2(b)).

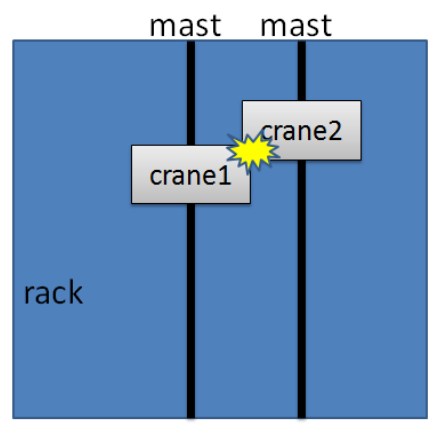

Front view

Pattern (a)

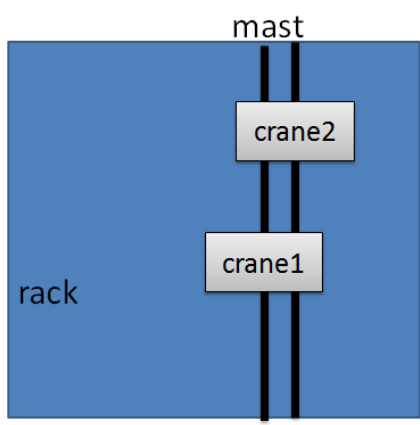

Front view

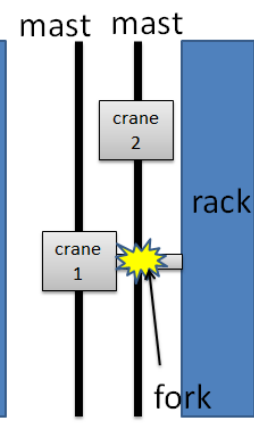

side view

Pattern (b)

Fig. 2 Collision conditions

\subsubsection{Limitation of stacker crane motion}

Since the movement of the stacker crane includes a vertical direction, we have to consider certain physical dynamics (e.g., vibration) and restrict the movement pattern. In other words, the trajectory of the crane cannot be modified once it has started to move. In addition, when a detour trajectory is adopted for avoiding collision, a via point is set to generate a detour trajectory, and the crane has to stop once at that point before moving to the destination.

\subsubsection{Trajectory selection and "normal trajectory"}

In addition, the control system for the horizontal and vertical direction is independent, 
which means horizontal and vertical movement is also independent. In Fig. 3, we focus on a similar situation, in which the crane is heading to its destination (indicated by an arrow). The velocity tables of the horizontal and vertical direction are generated according to the horizontal distance and vertical distance. In this instance, the movement time for the vertical direction is shortened more than that of the horizontal time, and the trajectory is shown as $a$. However, it is possible to move the Y-velocity table along the time-axis while maintaining the form of the table. The trajectory of the crane will be modified according to changes in the velocity tables. For example, in Fig. 4, if we move the start time of the movement from 0 to $t$ in the vertical direction, the trajectory of the crane will change from a to $b$, in which the movement time of $a$ and $b$ is the same.

Trajectories a and b are defined as "outer trajectories," and those between the outer trajectories are defined as "inner trajectories." There are infinite theoretical trajectories between the start and target positions (Fig. 5). However, it is easy to understand that, if both of the outer trajectories ( $a$ and $b$ ) collide with the trajectory of the other crane, inner trajectories cannot avoid collision. Therefore, if we want to find a non-collision trajectory, checking outer trajectories would be sufficient. The outer trajectories are defined as a "normal trajectory" in this paper.

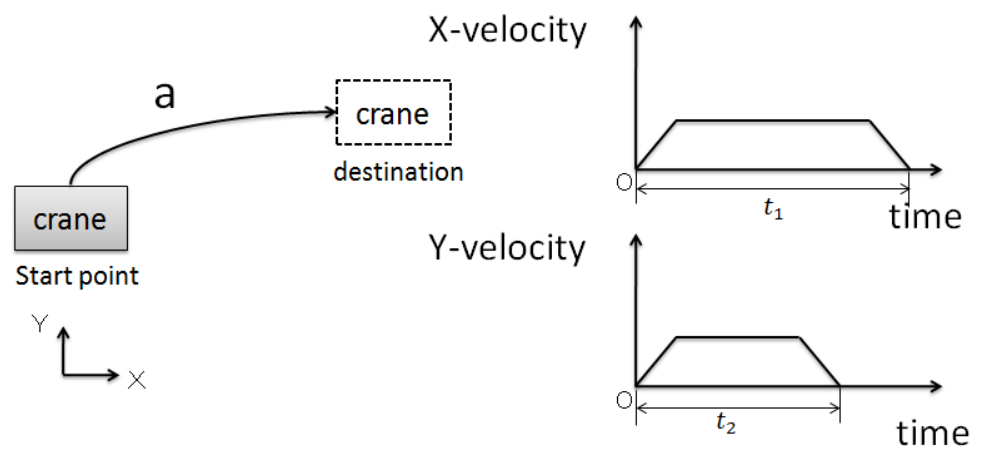

Fig. 3 Crane trajectory and velocity timetables for each direction

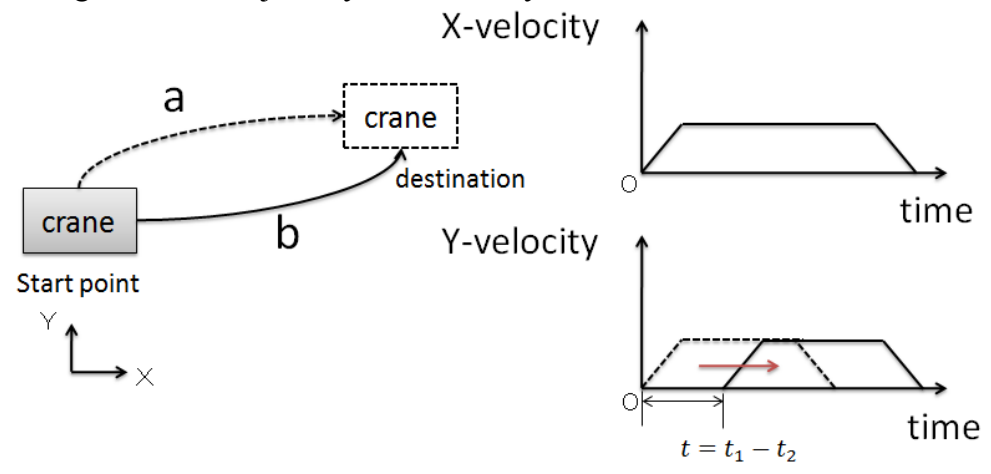

Fig. 4 Altered crane trajectory and velocity timetables for each direction 


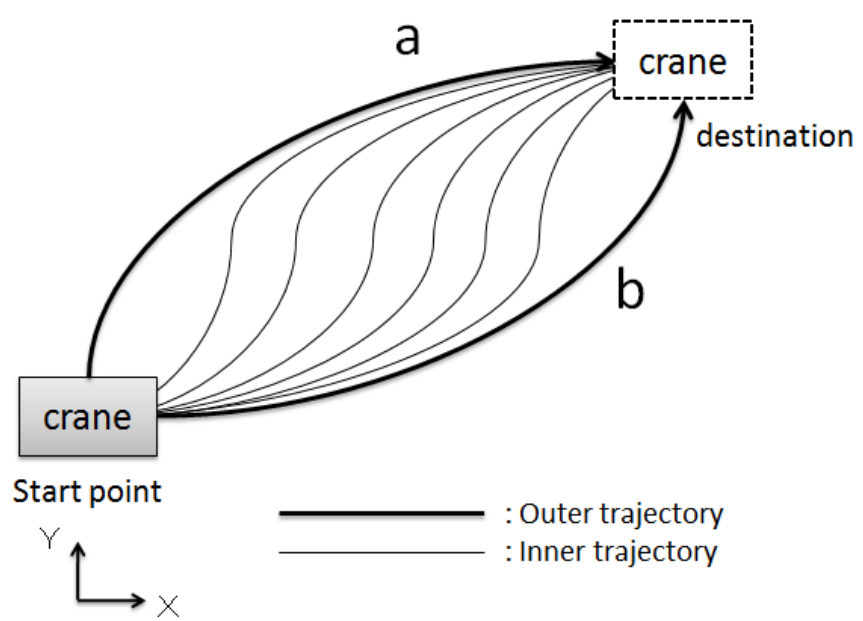

Fig. 5 Possible trajectories

\subsection{Motion planning method proposed in a previous study}

\subsubsection{Flowchart of motion planning}

Generally, the normal trajectories are the fastest option toward a destination. However, avoidance trajectories must be planned if cranes collide in the normal trajectory. The flowchart of motion planning is shown as Fig. 6.

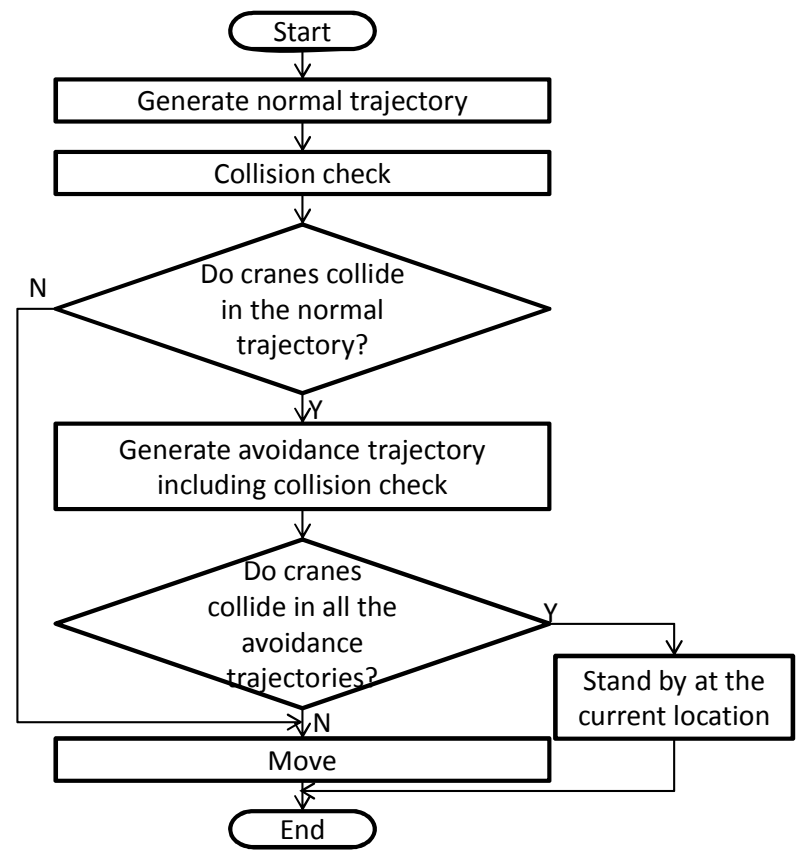

Fig. 6 Flowchart of motion planning in the former study

In the former study [21], collision patterns were examined, and two methods to generate efficient avoidance trajectories were developed. The proposed methods are as follows:

\subsubsection{Step 1: To delay movement in one direction}

This method is used when the collision is avoidable if we delay the crane for a given duration, as in Fig. 7. One crane (crane 1) is trying to organize an appropriate trajectory to its destination. In the meantime, the other crane (crane 2) is moving toward its destination, 
and, thus, the trajectory cannot be modified because of mechanical limitations. In this situation, if crane 1 goes straight to its destination, it will collide with crane 2. However, the collision can be avoided if crane 1 is delayed for a given duration.

In addition, the appropriate delay time for crane 1 is determined by using binary search to find the shortest delay time with which there will be no collision with the other crane.

\subsubsection{Step 2: Creating a detour}

This method is used if collision is inevitable in step 1. As shown in Fig. 8, crane 1 is attempting to organize an appropriate trajectory to its destination. In this situation, no matter how long movement is delayed by crane 1, collision cannot be avoided. Therefore, a via point will be set on the line passing through the collision point and normal to the original trajectory of crane 1 , and a new trajectory, which redirects to the via point, will be generated to avoid collision.

In addition, to find an appropriate via point, we draw a line normal to the normal trajectory and pass through the potential collision point and then conduct binary search on this line to find the via point that is the nearest to the potential collision point.

In this research, we will use [21]'s method to generate avoidance trajectories and focus on the calculation time for collision avoidance verification.

\subsubsection{Conduct collision check}

For each trajectory that is generated, we have to conduct a collision check to verify whether or not it is usable. Trajectories will not be considered as a candidate if a collision occurs.

To conduct a collision check, we calculate the crane's location of every single step (sampling time) and check to determine if it overlaps with another crane's location at the same step until the end of the trajectory (Algorithm 1) (Fig. 9).

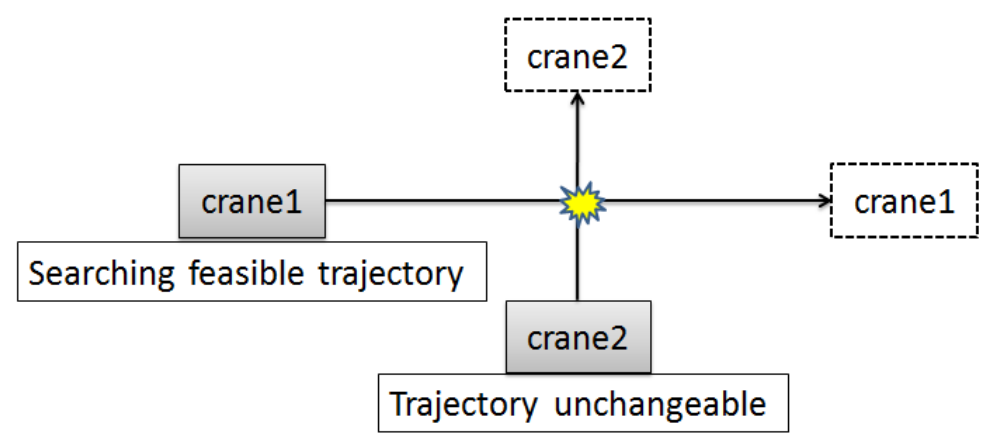

Fig. 7 A situation in which movement is delayed in one direction

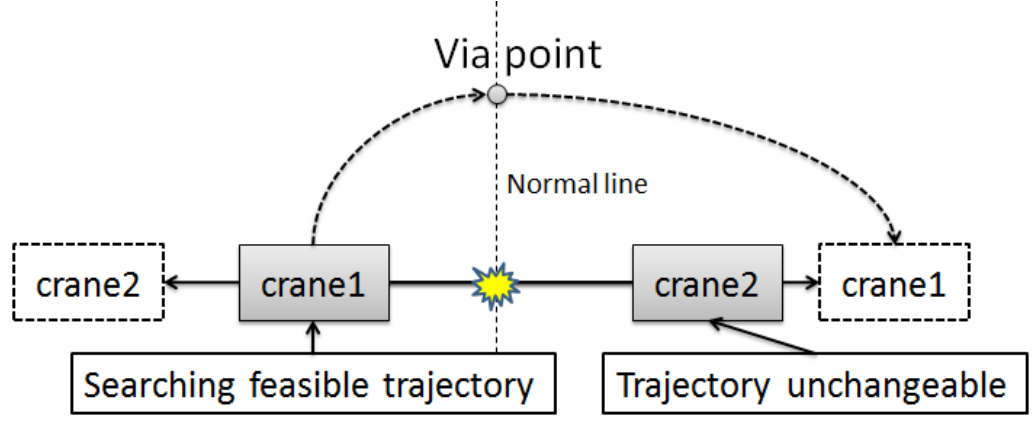

Fig. 8 A situation in which a detour is made 


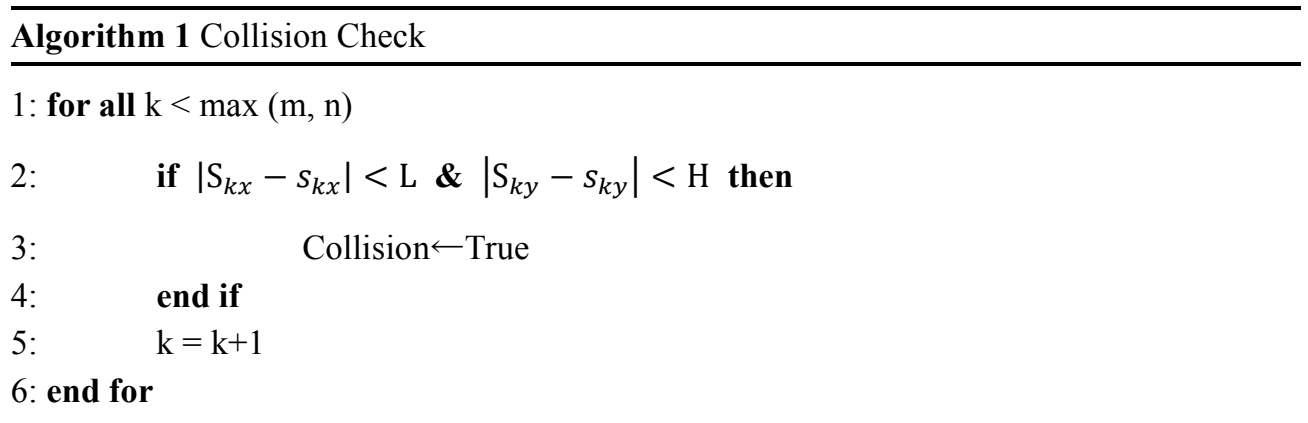

$\mathrm{m}$ : last trajectory step point of crane 1 .

$\mathrm{n}$ : last trajectory step point of crane 2 .

$\mathrm{S}_{k}$ : k-th trajectory step point of crane 1.

$\mathrm{s}_{k}: \mathrm{k}$-th trajectory step point of crane 2.

$\mathrm{S}_{k x}: \mathrm{X}$-coordinate of $\mathrm{S}_{k}$.

$\mathrm{S}_{k y}:$ Y-coordinate of $\mathrm{S}_{k}$.

$\mathrm{s}_{k x}: \mathrm{X}$-coordinate of $\mathrm{s}_{k}$.

$\mathrm{s}_{k y}:$ Y-coordinate of $\mathrm{s}_{k}$.

L: Length of crane.

H: Height of crane.

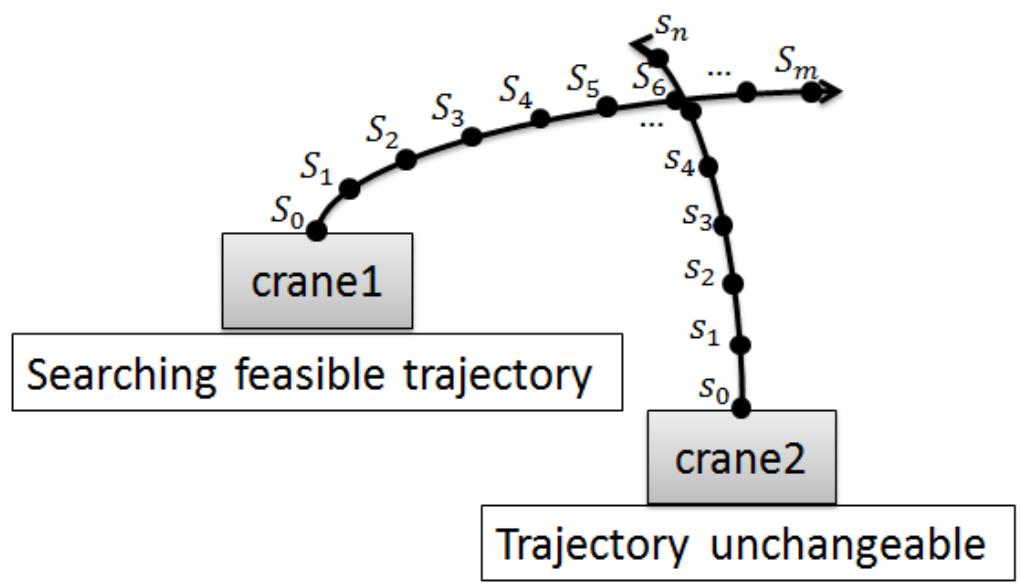

Fig. 9 Standard process of collision check

\subsection{Motion planning time and working time formulation}

The motion planning time and work time can be formulated as follows:

$\forall L_{i} \in$ Normal trajectories and trajectories generated in 2.2.2. and 2.2.3.

$$
t_{k}=\sum_{i=0}^{n u m b e r} \text { of trajectory candidates } L_{i}
$$

$L_{i}$ : Collision avoidance verification time for i-th trajectory candidate.

$t_{k}$ : Motion planning time for $\mathrm{k}$-th movement task.

In addition, the time for the completion of a warehouse task is shown as:

$$
\begin{array}{r}
T_{k}=f\left(E_{k-1}, S_{k}\right)+f\left(S_{k}, E_{k}\right)+d_{k} \\
\quad \text { All task completion time }=\sum T_{k}
\end{array}
$$

$T_{k}$ : Moving time of $\mathrm{k}$-th task.

$S_{k}$ : k-th start position.

$E_{k}$ : k-th end position.

$\mathrm{f}\left(P_{m}, P_{n}\right)$ : Movement time from $P_{m}$ to $P_{n}$. 
$d_{k}$ : Standby time and loading time on k-th task.

While the quality of trajectory $\left(\sum T_{k}\right)$ is satisfied in the previous study, it takes too much calculation time $\left(t_{k}\right)$ to generate an appropriate trajectory. In this paper, we propose a method for the rapid planning of a trajectory that can be used in an online environment; this means that we want to suppress all $t_{k}$ to below $0.1 \mathrm{~s}$. Although we reduce the calculation time in motion planning, we also give attention to maintaining comparable efficiency to that achieved in the previous study.

\section{Proposed method}

The outline of the proposal is shown in Fig. 10. First, we generate normal trajectories; if, in the normal trajectories, one crane collides with another, we then generate avoidance trajectories, as reported in Section 2.2. While the generation of trajectories is done very fast, it takes considerable computational time to check for collisions with other cranes for each generated trajectory. This is particularly true when normal trajectories are unable to avoid collision and numerous avoidance trajectory candidates have to be generated to find an appropriate avoidance trajectory. As collision avoidance verification is required for every trajectory candidate in order to find a feasible route, a great deal of computational time is required. For reducing the calculation time of motion planning, we intend to undertake it from two aspects. One is to reduce the number of collision checks by expanding the collision check period. The other is to arrange the collision check sequence in order to more quickly identify an appropriate trajectory.

These two improvements (written in red characters in Fig. 10) are specifically described as Section 3.1 and Section 3.2.

\subsection{The utilization of a "free-step"}

In the previous study, to carry out collision avoidance verification for a candidate trajectory, both a collision check and, if the current task was completed, planning for subsequent motion were executed for every single discrete time (step) (Fig. 9). There are more sampling points to be checked for collision in a long-distance move. This means that the computational time for collision checks of a candidate trajectory $L_{k}$ is proportional to the length of the trajectory. Therefore, it will take a great deal of time to carry out the collision check in a large-scale warehouse. In this paper, we propose a "free-step" to check for collision only when a crane might collide with another instead of checking for collision at every sampling time. The free-step is the number of steps (sampling time) that a crane can take without colliding with another. We calculate the time span in which cranes will not collide with each other as they move. The derivation of the free-step is to calculate separately the horizontal direction free-step and vertical direction free-step based on the formula shown below. According to the conditions of "the velocity and direction of each crane" and "whether or not the speed of the crane attains maximum" (Fig. 11), we can derive the minimum time span to avoid collision. For example, as shown in formula (5) and Fig. 11, the direction of $V_{1}$ and $V_{2}$ is the same. We want to calculate the time span during which crane 1 can move without a collision check; thus, we consider an extreme situation in which crane 2 moves very slowly and its velocity is near 0 and crane 1 moves to the right at maximum horizontal velocity. In this situation, the time span in which a crane can move without collision will be the division of $\mathrm{dx}$ and the maximum horizontal velocity. Other formulas can be derived by the same method.

For the free-step of the $\mathrm{x}$ direction, 


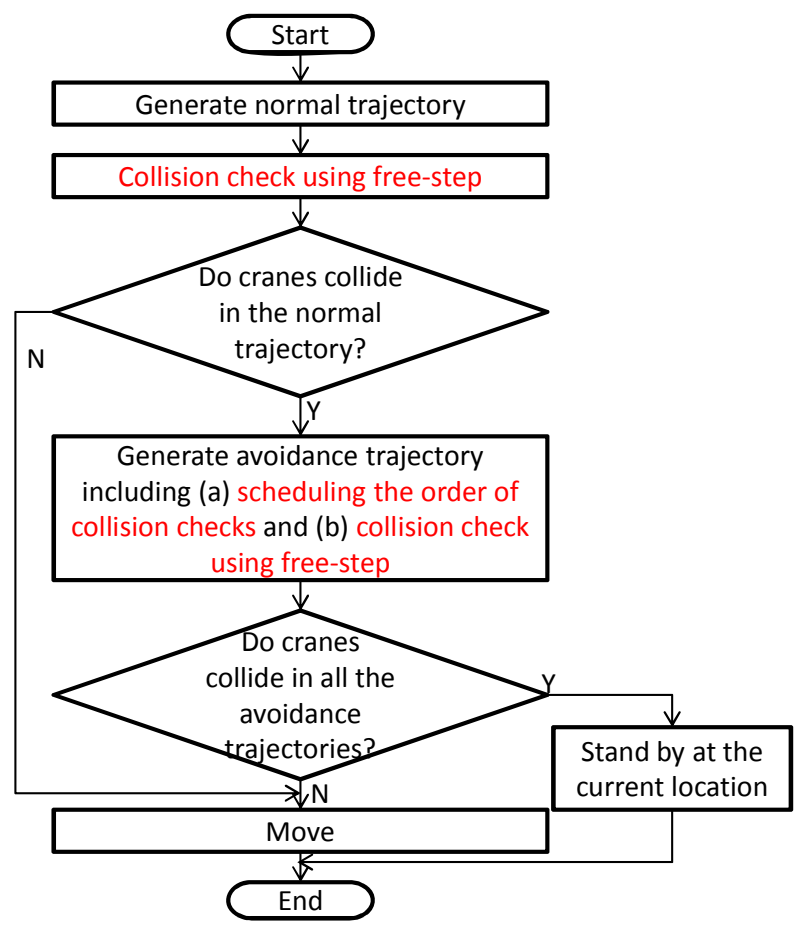

Fig. 10 Flowchart of collision avoidance verification

X_free-step $=\{$

$$
\begin{aligned}
& \frac{d x}{2 V_{\max }}\left(V_{1} \cdot V_{2}<0\right) \\
& \frac{d x}{V_{\max }}\left(V_{1} \cdot V_{2}>0\right) \\
& \sqrt{\frac{V_{\max }{ }^{2}+2 a \cdot d x}{a}}\left(V_{1} \cdot V_{2}=0, V_{1}+V_{2} \neq 0, \mathrm{dx}<\frac{V_{\max }{ }^{2}}{a}\right) \\
& \frac{d x}{2 V_{\max }}+\frac{V_{\max }}{4 a}\left(V_{1} \cdot V_{2}=0, V_{1}+V_{2} \neq 0, \mathrm{dx} \geq \frac{V_{\max }{ }^{2}}{a}\right) \\
& \sqrt{\frac{d x}{a}}\left(V_{1}=V_{2}=0, \mathrm{dx}<\frac{V_{\max }{ }^{2}}{a}\right) \\
& \frac{V_{\max }{ }^{2}+a \cdot d x}{2 V_{\max } \cdot a}\left(V_{1}=V_{2}=0, \mathrm{dx} \geq \frac{V_{\max }{ }^{2}}{a}\right)
\end{aligned}
$$

a: acceleration of $\mathrm{x}$ direction.

$V_{\max }$ : maximum velocity of $\mathrm{x}$ direction.

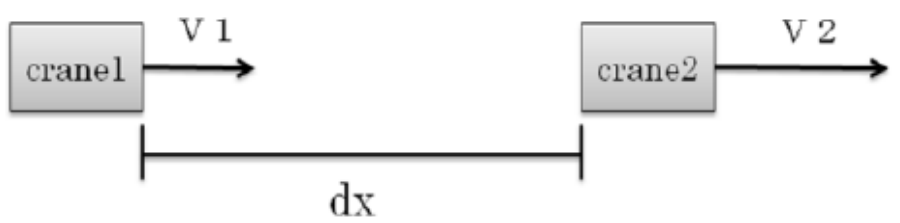

Fig. 11 Velocity and distance relation of the $\mathrm{x}$-axis between cranes

The free-step of the $y$-direction, $Y$ free-step, can be derived with similar formulas by replacing $\mathrm{x}$ with $\mathrm{y}$. Since the crane collides only when both the $\mathrm{x}$ and $\mathrm{y}$ positions overlap, the free-step will be the larger of the $X_{-}$free-step and $Y_{-}$free-step.

$$
\text { free-step }=\max (X \text { free-step, } Y \text { free-step }) \text {. }
$$


The proposed method will calculate the free-step every time before a collision check and then move free-step steps rather than only one step to do the collision check. (Algorithm 2).

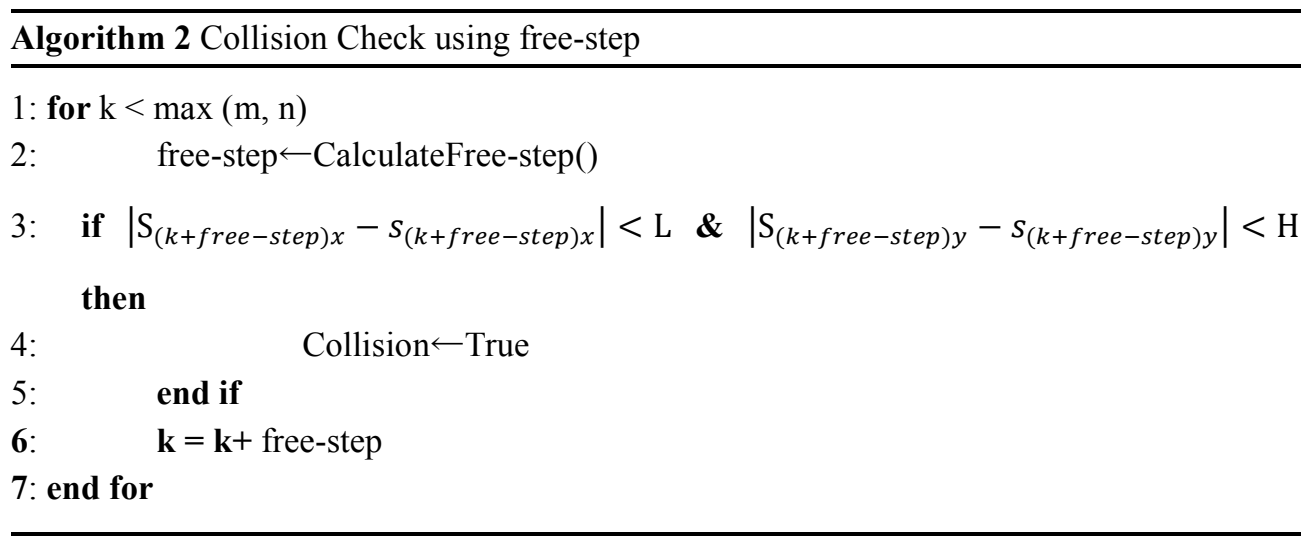

CalculateFree-step (): Calculate appropriate free-step shown as (11).

\subsection{Schedule the order of collision checks}

For a set of start and target positions, there is more than one possible trajectory. For those numerous possible trajectory candidates, collision verification is conducted, and the trajectory with the shortest moving time is then chosen as the solution. As there are a considerable number of candidates for an appropriate trajectory, extended time can be required if collision avoidance verification is calculated randomly. Therefore, it is important to schedule the order of the collision checks.

In earlier research [21], collision checks for possible trajectories were executed randomly. However, when collision avoidance verification occurs, we have found that some trajectory candidates have a high probability of collision, and some do not. If we ignore this factor and collision avoidance verification is done randomly, it may take a considerable amount of time to check all feasible trajectories.

In this paper, we propose an efficient method to schedule the order of collision checks for a given trajectory. The trajectory with a higher probability for collision avoidance should be checked first, and, thus, the time for collision check can be reduced. We use the location of two cranes and choose a trajectory that is more distant from the other crane in a normal trajectory situation. For instance, in Fig. 12, crane 2 is moving to its destination, and its trajectory is unchangeable. crane 1 generated normal trajectories $T_{1}$ and $T_{2}$ for movement. Here, since $T_{1}$ is more distant from crane 2, we consider $T_{1}$ as having a higher possibility of avoidance than $T_{2}$, and we do collision avoidance verification of $T_{1}$ first. In a detour avoidance trajectory situation, the previous study set several via points based on the expected collision point. For each via point, there are four possible avoidance trajectories. For example, in Fig. 13, the possible trajectories could be the combination of a trajectory from a start point to a via point and that from a via point to a destination, which are $T_{3} T_{5}, T_{3} T_{6}, T_{4} T_{5}$, and $T_{4} T_{6}$. The collision check for those trajectory candidates was performed randomly. However, in this paper, we only carry out a collision check on the trajectory that is farthest from the expected collision point, which is $T_{3} T_{5}$ in Fig. 13 . 


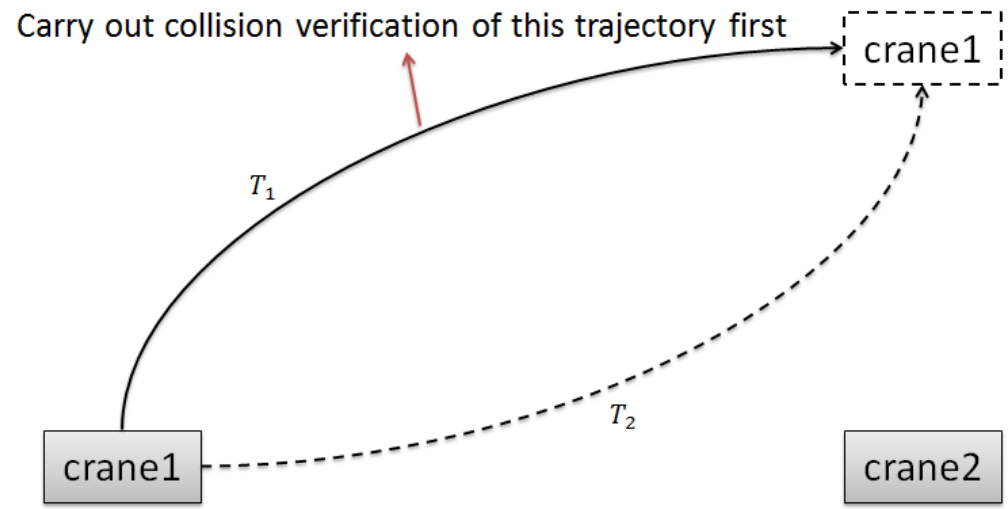

Fig. 12 Derivation of the order of collision avoidance verification in a normal trajectory

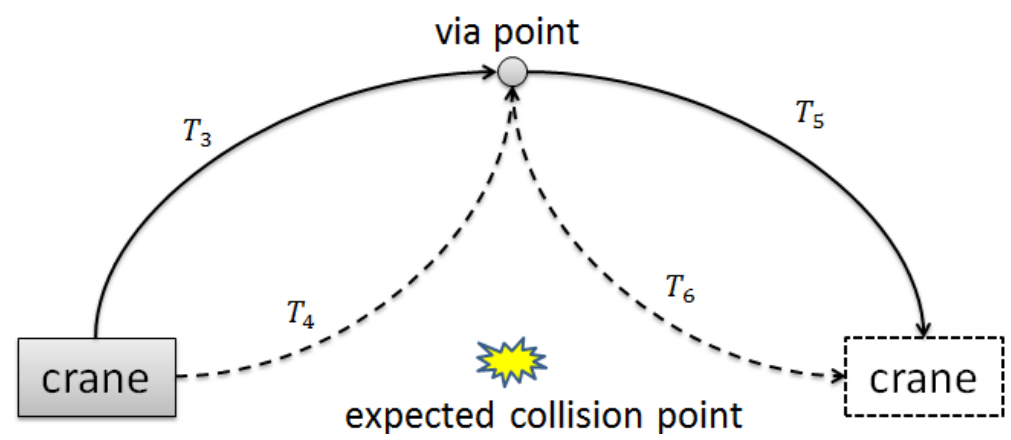

Fig. 13 Derivation of the order of collision avoidance verification in a detour avoidance trajectory

To derive the farthest trajectory from the trajectory candidate, we draw a line between the start position and the target position and calculate its equation (Fig. 14); we can then understand whether the point we want to avoid is on the left side or the right side of the line by substituting the location of the point into the line equation and then choosing the trajectory which is on the opposite side (Table 1).

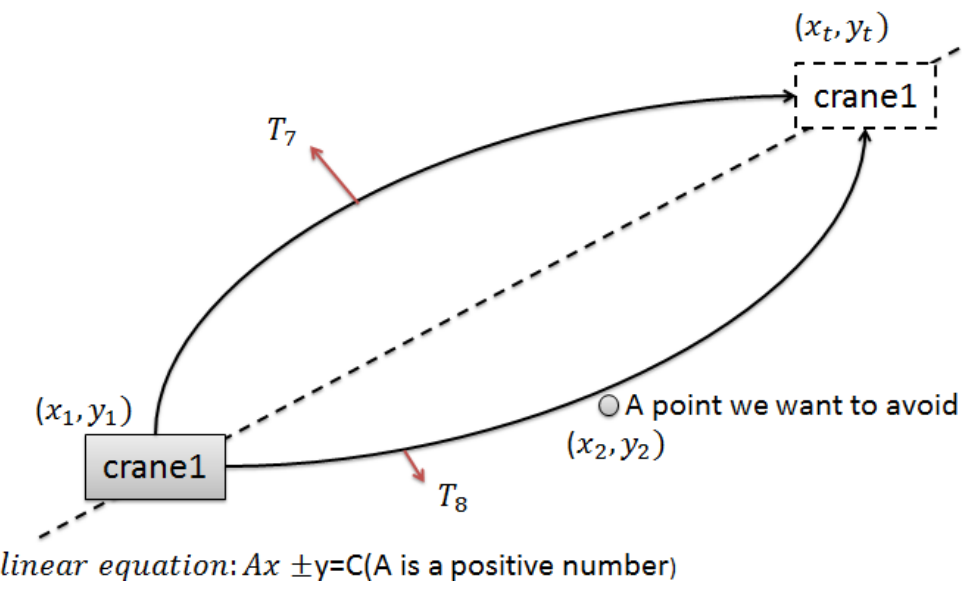

Fig. 14 Derivation method of the farthest trajectory 
Table 1. Table to derive the farthest trajectory

\begin{tabular}{|c|c|c|}
\hline & $y_{t}-y_{1} \geq 0$ & $y_{t}-y_{1}<0$ \\
\hline$A x_{2} \pm y_{2}-C \geq 0$ & $T_{7}$ & $T_{8}$ \\
\hline$A x_{2} \pm y_{2}-C<0$ & $T_{8}$ & $T_{7}$ \\
\hline
\end{tabular}

\section{Simulations}

A computer with an Intel Xeon $3.0 \mathrm{GHz}$ CPU with 2044MB of memory was used to carry out the simulation. Since the objective of this paper is to show a method of providing a motion planning time of $0.1 \mathrm{~s}$ using a normal industrial computer (with a $2.0 \mathrm{GHz} \mathrm{CPU}$ ), we have to modify our objective to $0.067 \mathrm{~s}$ per motion planning time due to the difference between the two computer processors.

In the warehouse simulation, we assigned 200 tasks randomly in an environment of 256 bays with 128 levels and another of 50 bays with 20 levels. We ran the simulations 5 times each, and we recorded the averages.

The simulation results are shown in Tables 2 and 3. "Working time" is the time consumed to finish all 200 tasks. The lower the working time duration, the better the work efficiency. Please be careful that the planning time is not included in the working time because planning for the next task is conducted during the time just before finishing the present task.

Table 2. Simulation results for a warehouse environment of 256 bays with 128 levels attempting 200 tasks

\begin{tabular}{|c|c|c|}
\hline & Previous study & Proposed method \\
\hline Working time (s) & $1.58 \times 10^{3}$ & $1.58 \times 10^{3}$ \\
\hline $\begin{array}{c}\text { Maximum calculation time for } \\
\text { collision avoidance verification (s) }\end{array}$ & 13.33 & 0.03 \\
\hline $\begin{array}{c}\text { The number of motion planning that } \\
\text { exceeds objective calculation time } \\
(0.067 \mathrm{~s}) \text { during 1000 time planning }\end{array}$ & 143.60 & 0 \\
\hline
\end{tabular}

Table 3. Simulation results for a warehouse environment of 50 bays with 20 levels attempting 200 tasks

\begin{tabular}{|c|c|c|}
\hline & Previous study & Proposed method \\
\hline Working time (s) & $5.66 \times 10^{2}$ & $5.73 \times 10^{2}$ \\
\hline $\begin{array}{c}\text { Maximum calculation time for } \\
\text { collision avoidance verification (s) }\end{array}$ & 3.30 & 0.01 \\
\hline $\begin{array}{c}\text { The number of motion planning that } \\
\text { exceeds objective calculation time } \\
(0.067 \mathrm{~s}) \text { during 1000 time planning }\end{array}$ & 33.40 & 0 \\
\hline
\end{tabular}

From the table, it is clear that the working time in the previous study and that in the proposed method are quite similar; however, the calculation time of motion planning of the proposed method is much less than that in the previous study. From the viewpoint of maximum calculation time for collision avoidance verifications, we can reduce the calculation time to approximately less than $1 / 300$ of that achieved in former research.

This is so because the proposed method saves a considerable amount of calculation time by utilizing a free-step, which is proportional to the distance between cranes. As the scale of the warehouse increases, the value of the free-step will also become larger; as a result, we can reduce the computational time of collision verification as the scale of the 
warehouse increases. On the other hand, since the computational time of collision verification is proportional to the distance of the crane's start point and end point in the previous study, as the scale of the warehouse increases, the computational time will also increase and exceed the time restriction in the warehouse industry. In fact, it is evident in Figs. 15 and 16 that, while the number of collision avoidance checks is proportional to the warehouse scale in the previous research, it becomes smaller when the warehouse scale increases and is not affected by the warehouse scale after the scale exceeds 100 , because, when the warehouse scale becomes larger, the free-step becomes larger as well. Consequently, the number of collision avoidance checks decreases. When the scale of the warehouse is larger than 100, the value of the free-step will be large enough for collision verification to be completed in a very short time. Therefore, for warehouse scales larger than 100 , the times of collision check will approach a small number.

If we only use the free_step algorithm to do the simulation, the largest calculation time of motion planning will be $0.08 \mathrm{~s}$ in a warehouse environment of 256 bays with 128 levels and $0.07 \mathrm{~s}$ in an environment of 50 bays with 20 levels. If we only use a reschedule order of the collision check algorithm to do the simulation, the largest calculation time of motion planning will be $0.54 \mathrm{~s}$ in a warehouse environment of 256 bays with 128 levels and $0.24 \mathrm{~s}$ in an environment of 50 bays with 20 levels. That means that the free_step algorithm is more influential than the reschedule order of the collision check algorithm here (Table 4).

Table 4. Comparison of proposed methods

\begin{tabular}{|c|c|c|c|}
\hline & Free_step only & $\begin{array}{c}\text { Reschedule order of } \\
\text { collision check only }\end{array}$ & $\begin{array}{c}\text { Both } \\
\text { algorithm } \\
\text { used }\end{array}$ \\
\hline $\begin{array}{c}\text { Maximum calculation time for } \\
\text { collision avoidance verification in } \\
\text { an environment of 50 bays with 20 } \\
\text { levels (s) }\end{array}$ & 0.07 & 0.24 & 0.01 \\
\hline $\begin{array}{c}\text { Maximum calculation time for } \\
\text { collision avoidance verification in } \\
\text { an environment of 256 bays with } \\
128 \text { levels(s) }\end{array}$ & 0.08 & 0.54 & 0.03 \\
\hline
\end{tabular}

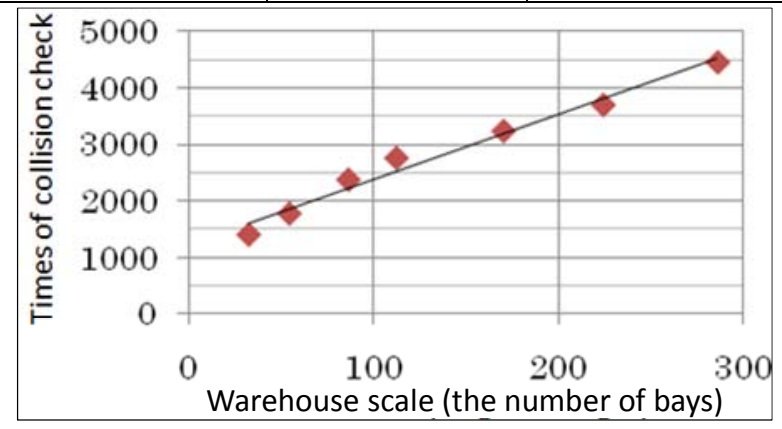

Fig. 15 Average number of collision check times per motion planning in the previous study 


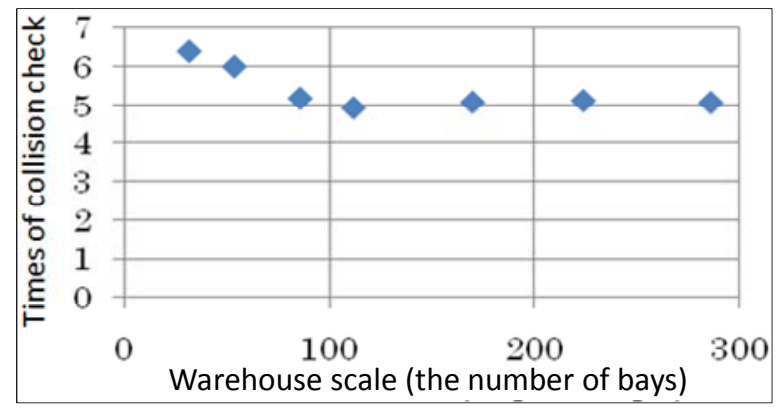

Fig. 16 Average number of collision check times per motion planning in the proposed method

\section{Conclusion}

Since AS/RSs are being used more frequently and the loading efficiency of warehouses has become important, a warehouse with two stacker cranes has been considered as a solution to improve the efficiency of AS/RSs. Although there are several studies about the motion planning method in a warehouse, the calculation time of motion planning is too long for application in the industrial field.

In this paper, we introduced two methods to reduce the calculation time of motion planning to a practical level. The first one is to utilize the free_step to cut down the redundant calculation time of collision verification. By utilizing this method, we can hold the calculation time even in a large-scale warehouse environment, which was a significant problem in the previous study. Furthermore, since the calculation time is irrelevant to the warehouse scale, the method is usable even if there is a much larger warehouse in the future. The second method is to reschedule the order of collision checks, which raises the possibility of finding an appropriate trajectory from numerous trajectory candidates faster.

By utilizing the proposed methods, the calculation time of motion planning is restricted to $0.067 \mathrm{~s}$, which is sufficiently small for use in an actual warehouse system, and the work efficiency is almost the same as that in the previous study.

We plan to promote the work efficiency by fully utilizing the remaining memory when planning avoidance trajectories.

\section{References}

[1] Kees, J.R., Iris, F.A., 2009: A survey of literature on automated storage and retrieval systems. European Journal of Operational Research Vol. 194, No. 2, pp.343-362.

[2] Tompkins, J.A., White, J.A., Bozer, Y.A., Tanchoco, J.M.A., 2003: Facilities Planning, $3^{\text {rd. }}$ Ed. John Wiley \& Sons.

[3] Malmborg, C.J., Bhaskaran, K., 1990: A revised proof of optimality for the cube-per-order index rule for stored item location. Applied Mathematical Modeling Vol. 14 No. 2, pp.87-95.

[4] Malmborg, C.J., Krishnakumar, B., 1989: Optimal storage assignment policies for multiday-dress warehousing systems. IEEE Transactions on Systems, Man and Cybernetics Vol. 19, No. 1, pp.197-204.

[5] Lee, M.K., 1992: A storage assignment policy in a man-on-board automated storage/retrieval system. International Journal of Production Research Vol. 30, No. 10, pp.2281-2292.

[6] Barrett, B.G., 1977: A further digression on the over-automated warehouse: Some evidence. Interfaces Vol. 8, No. 1, pp.46-49.

[7] Elsaed, E.A., 1981: Algorithms for optimal material handling in automatic warehousing systems. International Journal of Production Research Vol. 19, No. 5, pp.525-535.

[8] Y.A Bozer, J.A. White, 1984: Travel-Time Models for Automated Storage/Retrieval Systems. 
IIE Transactions Vol. 16, No. 4, pp.329-338.

[9] M.H. Han, L.F. McGinnis, J.S. Shieh, J.A. White, 1987: On Sequencing Retrievals in an Automated Storage/Retrieval System. IIE Transactions, pp.56-66.

[10] Heungsoon Felix Lee, Samantha K. Schaefer, 1997, Sequencing Methods for automated storage and retrieval systems with dedicated storage, Computers \& Industrial Engineering, 32 (2), 351-362.

[11] M. Eben-Chaime, 1992: Operations sequencing in automated warehousing systems. International Journal of Production Research 30 (10), 2401-2409.

[12] E.A. Elsayed, Unal, 1989: Order Batching Algorithms and Travel Time Estimation for Automated Storage/Retrieval Systems. International Journal of Production Research, Vol. 27, No. 7, pp.1097-1114.

[13] Eynan, A., Rosenblatt, M.J., 1993: An interleaving policy in automated storage/retrieval systems. International Journal of Production Research Vol. 31, No. 1, pp.1-18.

[14] Eynan, A., Rosenblatt, M.J., 1994: Establishing zones in single-command class-based rectangular $A S / R S$. IIE transactions Vol. 26, No. 1, pp.38-46.

[15] Fohn, S.M., Greef, A.R., Young, R.E., O’Grady, P.J., 1994: A constraint system shell to support concurrent engineering approaches to design. Artificial Intelligence in Engineering 9 (1), 1-17.

[16] Foley, R.D., Frazelle, E.H., 1991: Analytical results for miniload throughput and the distribution of dual command travel time. IIE Transactions Vol. 23, No. 3, pp.273-281.

[17] Heskett, J.L., 1963: Cube-per-order index - a key to warehouse stock location. Transportation and Distribution Management Vol. 3, pp.27-31.

[18] Heskett, J.L., 1964: Putting the cube-per-order index to work in warehouse layout. Transportation and Distribution Management 4, 23-30.

[19] Eben-Chaime, M., Pliskin, N., 1996: An integrative model for automatic warehousing systems. International Journal of Computer Integrated Manufacturing 9 (4), 286-292.

[20] Eben-Chaime, M., Pliskin, N., 1997: Operations management of multiple machine automatic warehousing systems. International Journal of Production Research 51, 83-98.

[21] H., Hino, Y., Kobayashi, T., Higashi, J., Ota, 2009: Control methodology of stacker cranes for collision avoidance considering dynamics in a warehouse. Proceedings of the 2009 IEEE International Conference on Robotics and Biomimetics, 983-988. 